\title{
Effets du tourteau des graines de Balanites aegyptiaca sur la consommation alimentaire et les performances zootechniques des cabris roux de Maradi
}

\author{
Adamou AKOURKI ${ }^{1 *}$, Abdoul Rachidou SODO DAKA ${ }^{2}$, Djibo IDRISSA SIDIKOU ${ }^{1}$ et \\ Issa CHAÏBOU ${ }^{1}$
}

\author{
${ }^{1}$ Enseignant Chercheur au Département des Sciences et Techniques d'Elevage, Université Dan Dicko \\ Dankoulodo de Maradi. Niger. \\ ${ }^{2}$ Etudiant en Licence Agronomie générale à l'Université Dan Dicko Dankoulodo de Maradi. Niger. \\ "Auteur Correspondant ; E-mail : adakna@yahoo.es; Tel : (+227) 20410132 /(+227) 91291868, Fax :
}

(+227) 20410133

\section{RESUME}

L'extraction d'huile contenue dans les amandes de Balanites aegyptiaca est un procédé industriel agroalimentaire qui ne cesse de se développer en Afrique sahélien mais qui génère à la fois une importante quantité de sous-produit tourteau, résultant souvent un encombrement pour les industries. Dans le cadre de la valorisation dudit sous-produit, le présent travail visait à évaluer les effets de l'incorporation du tourteau de Balanites aegyptiaca dans la ration sur les performances zootechniques des cabris roux de Maradi. Il a porté sur 12 cabris de race rousse de Maradi, âgés en moyenne de 7,5 10,5 mois, répartis en deux lots de 6 cabris chacun : un lot expérimental comprenant les cabris recevant une ration complémentée en tourteau de Balanites aegyptiaca (E) et un lot témoin constitué des cabris $(\mathrm{T})$ nourris à base de l'alimentation communément distribuée aux chèvres dans la région de Maradi. Les paramètres suivis étaient entre autres : la quantité d'aliments ingérés, le gain de poids et le rendement après abattage. Les moyennes des quantités d'aliments

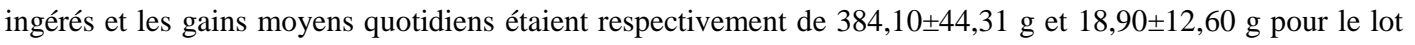
$\mathrm{E}$, et de $349,61 \pm 89 \mathrm{~g}$ et de $10,74 \pm 11,99 \mathrm{~g}$ pour le lot $\mathrm{T}$. Les $\mathrm{p}$ values correspondantes étaient de 0,000 et 0,001 , respectivement pour la quantité d'aliment ingérée et le gain de poids. Quant aux rendements carcasse et peau, ils étaient respectivement, de $43,18 \pm 0,74 \%$ et $8,22 \pm 1,57 \%$ pour le lot $\mathrm{E}$ et de $36,36 \pm 4,42 \%$ et $7,07 \pm 1,09 \%$ pour le lot $\mathrm{T}$ sans différence significative entre les lots $(\mathrm{p}>0,05)$. Cette expérience montre que le tourteau des graines de Balanites aegyptiaca n'a pas permis d'améliorer les rendements des cabris, il peut toutefois être utilisé par les éleveurs sahéliens pour augmenter la qualité de la ration en association avec la paille naturelle distribuée régulièrement aux animaux pendant la période sèche. Par ailleurs, une étude portant sur différentes concentrations de tourteau de Balanites aegyptiaca permettra sans doute d'optimiser le taux dudit tourteau dans la ration alimentaire des petits ruminants.

(C) 2019 International Formulae Group. All rights reserved.

Mots clés : Cabris roux de Maradi, performances zootechniques, tourteau, Balanites aegyptiaca et aliment bétail. 


\title{
Effects of Balanites aegyptiaca seed cake on food consumption and zootechnical performance of Maradi red goats
}

\begin{abstract}
The extraction of oil contained in the almonds of Balanites aegyptiaca is an agri-food industrial process that is being developed in Sahelian Africa but which generates a large amount of by-product cake, often resulting in congestion for industries. In the framework of valorization of this by-product, the present work aimed to evaluate the effects of the incorporation of Balanites aegyptiaca seed cake in the diet on the zootechnical performance of Maradi red goats. It involved 12 Maradi red-skinned goats, aged on average $7.5 \pm$ 0.5 months, divided into two lots of 6 kids each: an experimental batch including goats fed by a supplemented meal of Balanites aegyptiaca (E) and a control group consisting of goats (T) fed by the diet commonly distributed to goats in the region of Maradi. The monitored parameters included: the amount of food ingested, weight gain and post-slaughter yield. The averages of the amounts of food ingested and the average daily earnings were $384.10 \pm 44.31 \mathrm{~g}$ and $18.90 \pm 12.60 \mathrm{~g}$ for lot $\mathrm{E}$, and $349.61 \pm 89 \mathrm{~g}$ and $10.74 \pm 11.99 \mathrm{~g}$ for lot $\mathrm{T}$, respectively. The corresponding p-values were 0.000 and 0.001 , respectively for the quantity of food ingested and the weight gain. The carcass and skin yields were respectively $43.18 \pm 0.74 \%$ and $8.22 \pm 1.57 \%$ for lot $\mathrm{E}$ and $36.36 \pm 4.42 \%$ and $7.07 \pm 1.09 \%$ for lot $\mathrm{T}$, without significant difference between batches ( $>>0.05)$. This experiment shows that Balanites aegyptiaca seed cake has not improved goat yields, but it can be used by Sahelian breeders to increase the quality of the ration in combination with natural straw regularly distributed to animals during the dry season. In addition, a study of different concentrations of Balanites aegyptiaca seed cake will probably make it possible to optimize the amount of this cake in the diet of small ruminants.
\end{abstract}

(C) 2019 International Formulae Group. All rights reserved.

Keywords: Maradi red goat, zootechnical performance, seed cake, Balanites aegyptiaca and livestock feed.

\section{INTRODUCTION}

En Afrique, la zone sahélienne constitue une des principales régions d'élevage par excellence. En effet, son bétail était estimé à 470957850 têtes d'animaux en 2017, soit $37,56 \%$ de l'effectif total du cheptel africain (FAO, 2018). Par ailleurs, la contrainte majeure de l'élevage dans le Sahel demeure le déficit alimentaire qui est exacerbé en période sèche pendant laquelle les aliments disponibles sont le plus souvent de moindre qualité. Néanmoins, des initiatives importantes locales sont souvent menées en vue d'augmenter le disponible alimentaire pour les animaux. Il s'agit entre autres de la culture fourragère (Malam Abdou et al., 2017a), de l'amélioration de la qualité des sous-produits agricoles en réduisant leur volume ou/et en leur additionnant un supplément protéique (Zoudi et al., 2005 ; Safoura, 2006 ; Kiema et al., 2008 ; Gnanda et al., 2015ab; Soumana et al., 2016; Malam
Abdou et al., 2017b ; Kindim, 2018). Pour le cas précis de la supplémentation protéique, plusieurs ingrédients ont été testés chez les petits ruminants. C'est le cas de l'utilisation des gousses de Piliostigma reticulatum, ainsi que du tourteau de coton (Elhadji Nouhou, 2014 ; Malam Abdou et al., 2017b). De nos jours, d'autres types de tourteaux sont en train de se produire grâce à la production de nouveaux types d'huiles végétales telles que l'huile de Balanites aegyptiaca, très riche en acides gras surtout palmitique, stéarique, oléique et linoléique (Mohamed et al., 2002). En effet, le Balanites aegyptiaca est un arbre dont l'aire de dissémination est très grande puisqu'on le rencontre en Afrique, Asie et Moyen Orient. Du point de vue usage socioéconomique, c'est une plante utilisée depuis longtemps par l'homme dans son alimentation, pour soigner certaines maladies et pour la cosmétique (Tiétiambou et al., 2015). Cette plante, grâce à ses feuilles et 
amandes, constitue également une ressource fourragère importante pour les ruminants surtout que les amandes sont très riches en protéines et huile. Mais, le tourteau obtenu de l'huile des amandes de Balanites n'est pas consommé par les humains à cause de son goût trop amer. Il est aussi peu utilisé comme aliment bétail au Niger, résultant ainsi un produit encombrant pour les industries agroalimentaires naissantes. La valorisation de ces tourteaux permettra alors de réduire les coûts de fabrication de l'huile.

Le présent travail s'inscrit donc dans le cadre de l'augmentation du disponible alimentaire pour bétail à travers la valorisation du tourteau de Balanites aegyptiaca par son intégration dans la ration des animaux. Il vise de manière spécifique l'évaluation de la consommation du tourteau de Balanites aegyptiaca ainsi que son effet sur les performances zootechniques des cabris roux de Maradi.

\section{MATERIEL ET METHODES}

L'étude a été conduite pendant trois mois (octobre 2017 à janvier 2018) au Centre Secondaire d'Élevage Caprin de Maradi (Niger, $7^{\circ} 41^{\prime}$ de longitude Est et $13^{\circ} 43^{\prime}$ de latitude Nord). Elle a consisté à comparer une ration témoin communément distribuée aux cabris dans la région de Maradi avec une ration expérimentale contenant du tourteau de Balanites aegyptiaca (Tableau 1). Pour cela, deux lots de cabris mâles; Lot $\mathrm{T}$ (témoin) et lot $\mathrm{E}$ (expérimental) âgés en moyenne de 7,5 $\pm 0,5$ mois et dont le poids vif moyen était de $12,25 \pm 1,4 \mathrm{~kg}$, ont été formés. Chaque lot était formé de manière aléatoire par 6 cabris roux de Maradi maintenus individuellement dans des loges de $2 \mathrm{~m}^{2}$. Chaque animal recevait une ration de $300 \mathrm{~g}$ d'aliment matin et soir avec un accès libre à l'eau. La composition chimique des ingrédients des différentes rations a été déterminée au laboratoire LANA /CERRA de Niamey-Niger (Tableau 2). Avant la distribution de chaque ration, les ingrédients ont été d'abord broyés et mélangés. Au début de l'expérimentation, tous les animaux ont été déparasités et vaccinés contre la pasteurellose.

Le dispositif expérimental était maintenu en place pendant une durée de trois mois, précédée d'une période de 2 semaines d'adaptation au régime alimentaire. A la fin de l'expérimentation, $50 \%$ des animaux appartenant à chaque lot ont été abattus.

Les paramètres évalués étaient les suivants :

$>$ Quantité moyenne quotidienne d'aliment ingéré ; elle est la différence entre la quantité moyenne d'aliment distribuée et le refus moyen quotidien de chaque lot d'animaux.

$>$ Gain moyen quotidien (GMQ) obtenu grâce à des pesées hebdomadaires de tous les cabris où GMQ du cabri = (poids final - poids initial) en g / durée de l'expérimentation en jours

$>$ Circonférence moyenne des testicules obtenue en mesurant toute les semaines le contour des deux testicules de chaque animal à l'aide d'un mètre ruban.

$>$ Rendement moyen carcasse (RMC) où rendement carcasse de l'animal $=$ (poids de la carcasse x 100) / poids vif de l'animal avant abattage.

$>$ Rendement moyen peau (RMP) où rendement peau de l'animal $=$ (poids de la peau x 100) / poids vif de l'animal avant abattage.

\section{Analyses statistiques}

Les données récoltées ont été saisies sur le tableur Excel avant de faire l'objet d'une analyse de variance à un facteur à l'aide du logiciel IBM SPSS statistic.20. Les moyennes obtenues ont été comparées entre elles par le test Chi carré au seuil de 5\%. 
Tableau 1 : Formulation des rations distribuées aux cabris.

\begin{tabular}{lcc}
\hline Ingrédients & Lot témoin & Lot expérimental \\
\hline Tourteau de Balanites aegyptiaca $(\%)$ & 0 & 27 \\
\hline Fane d'arachide (\%) & 13,7 & 0 \\
\hline Paille de brousse (\%) & 64 & 64 \\
\hline Son de blé (\%) & 13,3 & 0 \\
\hline Sel (\%) & 3 & 3 \\
\hline Calcaire $(\%)$ & 6 & 6 \\
\hline Total $(\%)$ & 100 & 100 \\
\hline
\end{tabular}

Tableau 2: Résultats des analyses bromatologiques des aliments utilisés pour la ration des animaux.

\begin{tabular}{lccccc}
\hline Aliment & $\begin{array}{c}\text { Matière } \\
\text { sèche } \\
(\boldsymbol{\%})\end{array}$ & $\begin{array}{c}\text { Matière } \\
\text { minérale } \\
(\boldsymbol{\%})\end{array}$ & $\begin{array}{c}\text { Cellulose } \\
\text { brute } \\
(\boldsymbol{\%})\end{array}$ & $\begin{array}{c}\text { Matière } \\
\text { azotée } \\
\mathbf{( \% )}\end{array}$ & $\begin{array}{c}\text { Extrait } \\
\text { éthéré } \\
\mathbf{( \% )}\end{array}$ \\
\hline Paille de brousse & $94 \pm 0,04$ & $4 \pm 0,15$ & $43 \pm 0,44$ & $2 \pm 0,04$ & 0,20 \\
\hline Son de blé & $92 \pm 0,06$ & $6 \pm 0,11$ & $15 \pm 0,56$ & $14 \pm 00$ & 1,26 \\
\hline Fane d'arachide & $93 \pm 0,14$ & $13 \pm 0,50$ & $30 \pm 0,03$ & $18 \pm 0,00$ & 2,10 \\
\hline $\begin{array}{l}\text { Tourteau de } \\
\text { Balanites } \\
\text { aegyptiaca }\end{array}$ & $95 \pm 0,05$ & $7 \pm 0,02$ & $8 \pm 0,78$ & $42 \pm 0,17$ & 8,28 \\
\hline
\end{tabular}

\section{RESULTATS}

L'analyse de variance a montré une différence statistiquement significative entre les deux groupes d'animaux au niveau de tous les paramètres étudiés sauf pour les variables rendements. En effet, la quantité moyenne d'aliment ingéré par cabri/jour était statistiquement plus importante $(\mathrm{p}=0,000)$ pour le lot expérimental soit $384,10 \pm 44,31 \mathrm{~g}$ contre 349,61 $\pm 44,89 \mathrm{~g}$ (Figure 1).

Une situation similaire a été observée pour les variables gain moyen quotidien en $\mathrm{g}$ $(\mathrm{p}=0,001)$ et circonférence testiculaire $(\mathrm{p}=0,000)$ des cabris où les valeurs moyennes obtenues étaient respectivement de $18,90 \pm 12,60 \mathrm{~g}$ et $18,09 \pm 0,96 \mathrm{~cm}$ et
$10,74 \pm 11,99$ g et $16,56 \pm 0,98 \mathrm{~cm}$ pour le lot expérimental et le lot témoin (Figures 2 et 3).

Par contre, les rendements carcasse et peau ne variaient pas de manière significative entre les deux lots d'animaux bien que la tendance fût des valeurs plus élevées chez le groupe des animaux recevant de tourteau de Balanites dans leur ration. Ainsi, des chiffres équivalents à $43,18 \pm 0,47 \%$ et $36,36 \pm 4,42 \%$ en rendement de carcasse et $8,22 \pm 1,57 \%$ et $7,70 \pm 1,09 \%$ en rendement de la peau ont été obtenus après abattage des cabris appartenant aux lots expérimental et témoin, respectivement. Les $\mathrm{p}$ values correspondantes obtenues entre les deux lots d'animaux étaient de 0,111 et 0,454 , respectivement pour les rendements carcasse et peau (Tableau 3). 


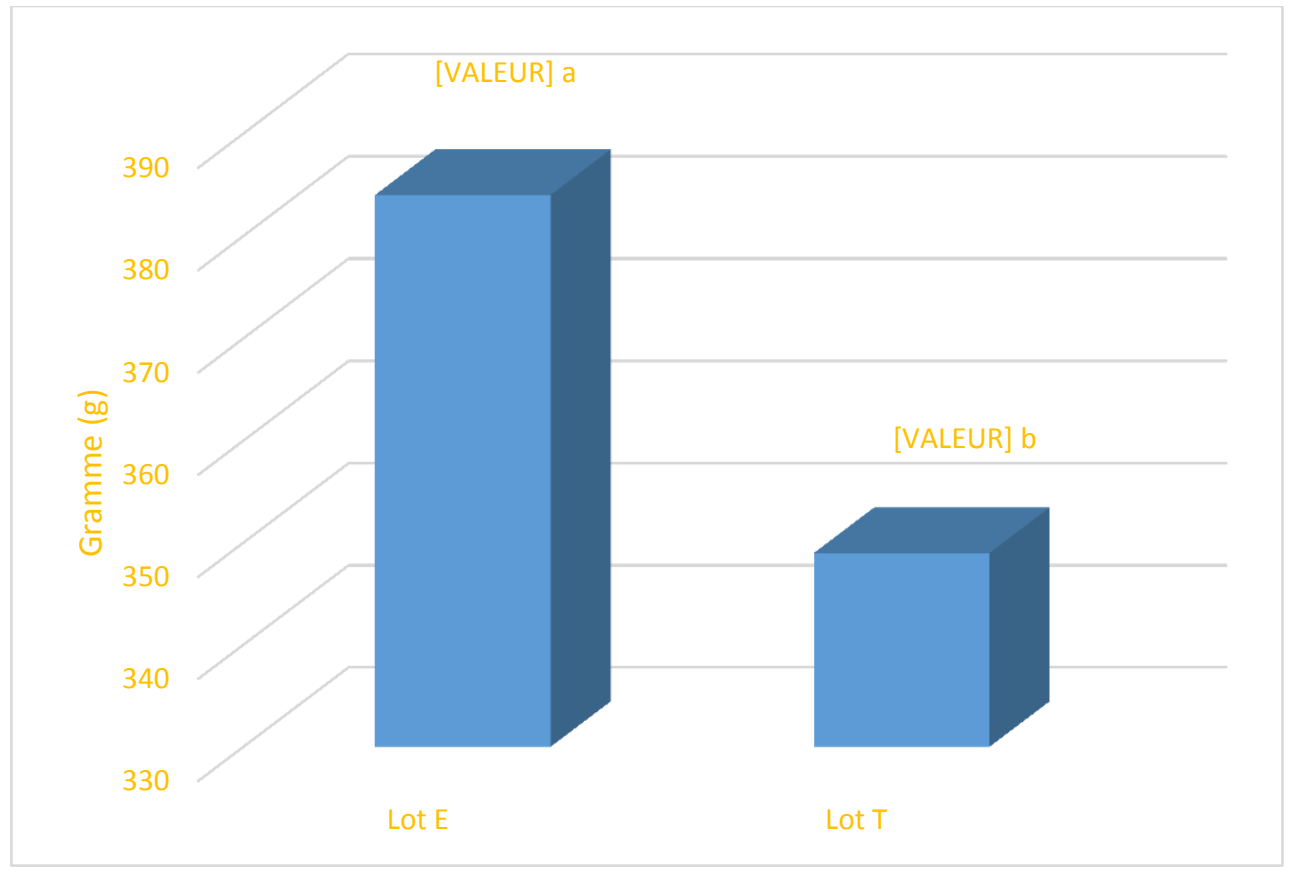

Les lettres $a$ et $b$ impliquent différence statistiquement significative $(p=0,000)$ entre les 2 lots d'animaux. Figure 1 : Quantité moyenne d'aliment ingéré en g par les différents lots de cabris.

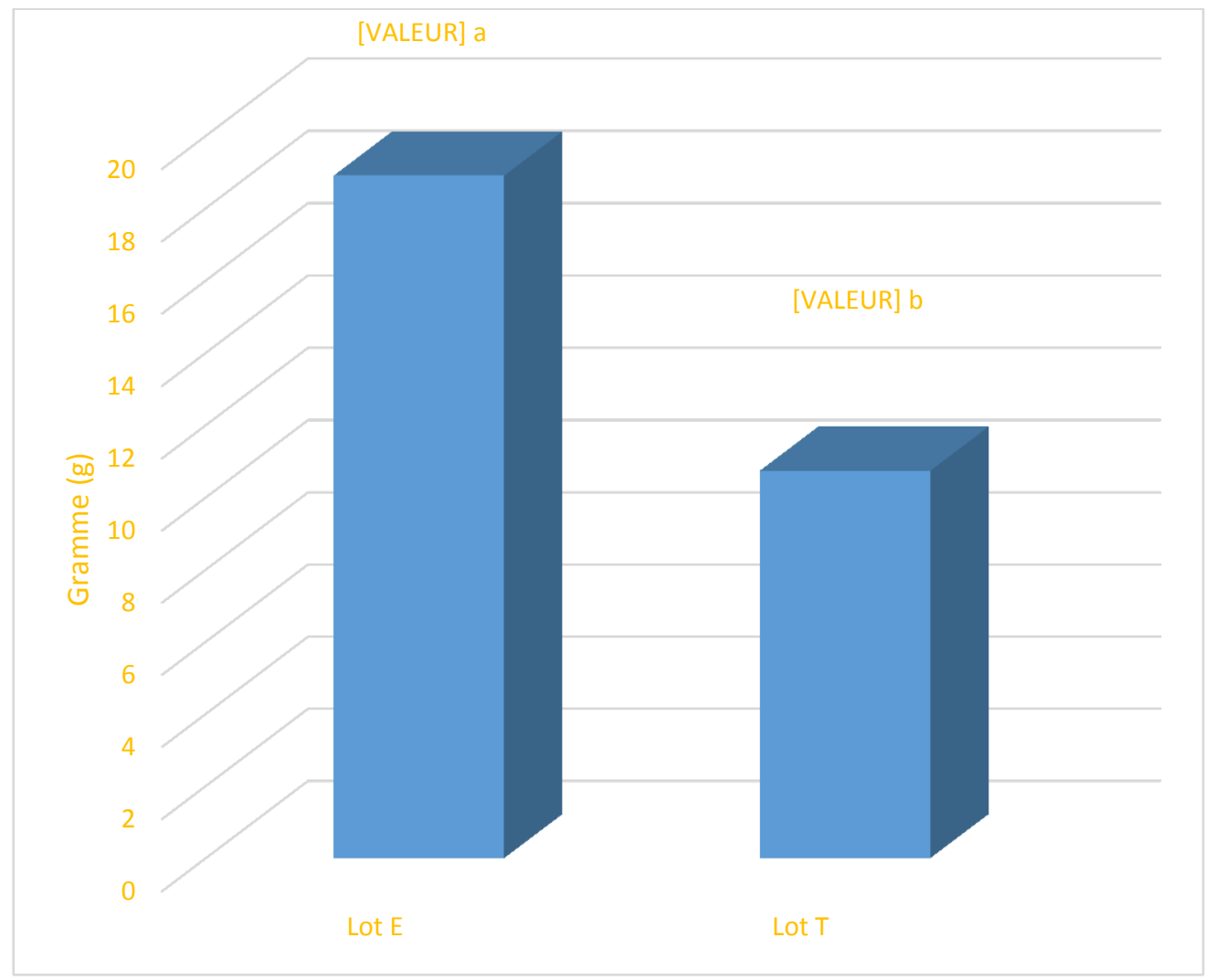

Les lettres a et $b$ impliquent différence statistiquement significative $(\mathrm{p}=0,001)$ entre les 2 lots d'animaux.

Figure 2 : Gain moyen quotidien en g (GMQ) obtenu par les lots de cabris. 


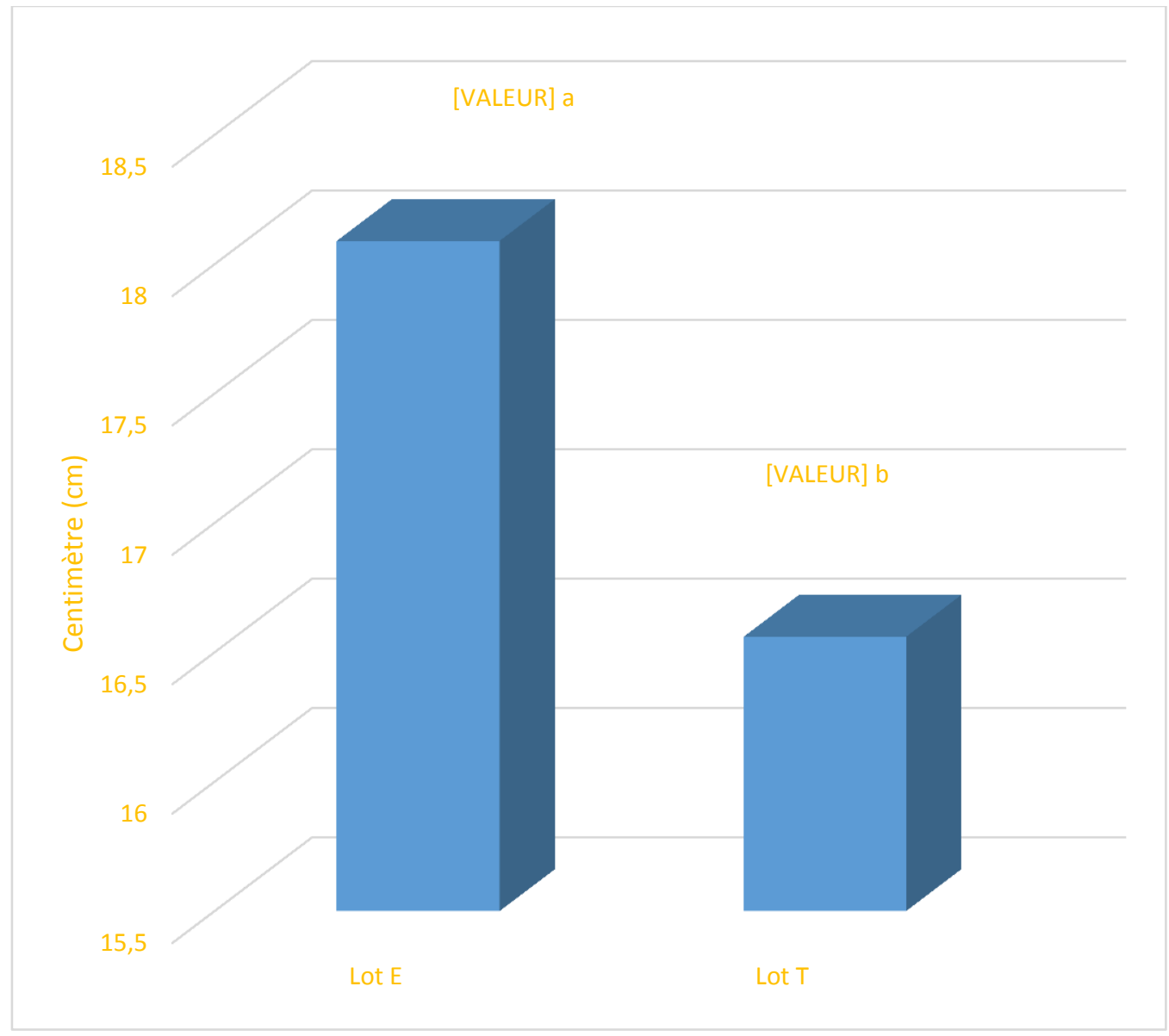

Les lettres a et $b$ impliquent différence statistiquement significative $(\mathrm{p}=0,000)$ entre les 2 lots d'animaux.

Figure 3 : Circonférence testiculaire moyenne en $\mathrm{cm}(\mathrm{CT})$ obtenu par les lots de cabris.

Tableau 3 : Rendements moyens carcasse et peau obtenus par les différents lots de cabris.

\begin{tabular}{lcc}
\hline & RC (\%) & RP (\%) \\
\hline Lot $\mathbf{E}$ & $43,18 \pm 0,47$ & $8,22 \pm 1,57$ \\
\hline Lot T & $36,36 \pm 4,42$ & $7,70 \pm 1,09$ \\
\hline P value & 0,111 & 0,454 \\
\hline
\end{tabular}

RC : Rendement moyen carcasse ; $\quad$ RP : Rendement moyen peau. 


\section{DISCUSSION}

Les résultats obtenus ont montré que les cabris alimentés avec la ration contenant le tourteau de Balanites ont consommé plus de nourriture, soit un surplus de $35 \mathrm{~g}$ en moyenne par animal par rapport au cabri du groupe témoin. Cette différence de quantité d'aliment ingérée pourrait être due à la granulométrie des deux rations formulées. En effet, suite au broyage des différents aliments contenus dans chaque ration, le tourteau de Balanites a présenté des particules de plus grandes tailles que la fane d'arachide et son de blé qui avaient un aspect plus fin. Cette situation pouvait entrainer une modification de la préhension chez les animaux d'une ration à une autre; ce qui sans doute a entrainé une plus grande consommation de la ration contenant de tourteau par les cabris en comparaison avec la ration du groupe témoin contenant essentiellement du son de blé et de la fane d'arachide. Par ailleurs, la quantité moyenne d'aliment ingérée par les cabris de la présente étude est moins importante que celle rapportée par Malam Abdou et al. (2017b). Ces auteurs ont obtenu une quantité de matière sèche équivalente entre $449,5 \mathrm{~g}$ et $549,2 \mathrm{~g}$ ingérée par animal/jour dans une étude portant sur l'analyse technicoéconomique des aliments densifiés sur les performances de croissances des boucs de race rousse de Maradi. Cette divergence pourrait s'expliquer par la différence d'âge ou du poids vif des animaux, et la composition nutritive des aliments utilisés pour nourrir les cabris. En effet, ces auteurs ont travaillé avec des cabris pesant 13 à $17 \mathrm{~kg}$ de poids, donc plus lourds que ceux du présent travail.

Par contre, les résultats de la présente étude sont similaires à ceux rapportés par Elhadji Nouhou (2014) et Kindim (2018) qui ont publié chez la même race une consommation moyenne par cabri respectivement, de $362,48 \mathrm{~g}$ à $390,83 \mathrm{~g}$ et de $353,45 \mathrm{~g}$ à $359,64 \mathrm{~g}$.

$\mathrm{S}$ 'agissant du gain moyen quotidien en $\mathrm{g}$, les cabris du lot expérimental ont montré également de meilleures performances avec une différence de 8,16 g en leur faveur. Ce qui est logique car la ration du lot expérimental était plus riche en protéines que celle du lot témoin ; soit un taux de matière azotée de $42 \%$ pour le tourteau de Balanites de la ration expérimentale contre $32 \%$ pour la combinaison fane d'arachide et son de blé de la ration du groupe témoin selon les résultats des analyses bromatologiques faites (Tableau 2). Le gain obtenu par nos animaux était sensiblement pareil à ceux de Elhadji Nouhou (2014) et Kindim (2018). En effet, dans une étude sur la substitution du tourteau de coton par le tourteau des gousses de Piliostigma reticulatum, Elhadji Nouhou (2014) a trouvé un gain moyen quotidien en g qui variait de $8,25 \mathrm{~g}$ à $24,28 \mathrm{~g}$ selon le taux de tourteau de Piliostigma reticulatum dans la ration des cabris roux de Maradi. Quant à Kindim (2018), il a rapporté un gain de poids de 6,21 $\mathrm{g}$ à $11,9 \mathrm{~g}$ dans une tentative de remplacement de la combinaison fane d'arachide et son de blé par la fane de niébé uniquement dans la ration des cabris roux. Par contre, le gain obtenu par nos animaux était plus bas que celui affiché par les cabris de même race dans l'étude conduite par Malam Abdou et al. (2017b). Ces auteurs ont rapporté un gain moyen quotidien de $29,8 \mathrm{~g}$ à $78,3 \mathrm{~g}$ selon la formule alimentaire analysée. Il est important de préciser que l'ensemble des cabris de la présente étude ont été victime d'un syndrome de diarrhée pendant une durée de 2 semaines même s'ils ont été traités par la suite. Ce qui pourrait ralentir leur croissance en comparaison avec les animaux de l'expérience de Malam Abdou et al. (2017).

L'analyse de la circonférence testiculaire a montré de même que les cabris du groupe expérimental ont eu la plus grande croissance des testicules, soit $1,53 \mathrm{~cm}$ de plus par rapport aux animaux du groupe témoin. Cette différence est logique puisque les cabris du groupe expérimental ayant eu le meilleur gain de poids, on pourra s'attendre qu'ils aient des testicules les plus développés car il existe une bonne corrélation entre la croissance de l'animal et le développement de ses organes 
génitaux (Ahmad et al., 2011 ; Moxon et al., 2015).

Concernant les rendements des cabris, la différence observée entre les deux lots d'animaux n'était pas significative. Ce qui laisse croire que l'apport nutritionnel supplémentaire aux animaux du groupe expérimental n'était pas suffisamment important pour permettre un dépôt important de la chaire entourée d'une peau bien développée en comparaison avec les cabris du lot témoin. Néanmoins, les rendements après abattage obtenus par nos cabris étaient semblables à ceux rapportés par la littérature sur la même race. Ainsi, Elhadji Nouhou (2014) avait obtenu un rendement moyen en carcasse de $36,28 \pm 1,39 \%$ chez des cabris alimentés à base de quatre taux de tourteau de gousses de Piliostigma reticulatum. Quant à Kindim (2018), il a rapporté des rendements de carcasse et de la peau équivalents respectivement à $36 \pm 2 \%-37 \pm 2 \%$ et $6 \pm 1,1 \%$ $6,1 \pm 1,1 \%$ pour des cabris alimentés à base de différents types de fanes.

\section{Conclusion}

Les cabris recevant du tourteau de graines de Balanites dans leur ration ont bien assimilé ce complément sans altération apparente dans leur état de santé. Mais cette assimilation du tourteau ayant permis aux cabris de croitre plus rapidement n'était pas en mesure d'assurer les meilleurs rendements post abattage. Néanmoins, le tourteau des graines de Balanites peut être utilisé par les éleveurs comme un complément protéique afin d'améliorer la qualité de la paille naturelle administrée aux animaux pendant la période sèche. Des études plus poussées notamment sur l'évaluation de différentes concentrations du tourteau de graines de Balanites permettront sans doute d'optimiser l'utilisation de ce complément dans l'alimentation des petits ruminants.

\section{CONFLIT D'INTERETS}

Les auteurs de ce manuscrit déclarent qu'il n'existe aucun conflit d'intérêt entre eux.

\section{CONTRIBUTIONS DES AUTEURS}

Cette étude a été possible grâce à la contribution de tous ses auteurs. En effet, AA a, non seulement élaboré le protocole de recherche et réalisé le traitement des données mais aussi a rédigé le manuscrit. RASD a assuré le suivi du dispositif expérimental et la collecte des données. DIS a participé à la collecte des données et à la relecture du manuscrit. Enfin, quant à IC, il a participé à l'élaboration du protocole de recherche et à la relecture du manuscrit.

\section{REMERCIEMENTS}

Les auteurs remercient énormément le Centre caprin de Maradi pour la disponibilité des installations, le Département de Sciences et Techniques d'Elevage de l'Université Dan Dicko Dankoulodo de Maradi pour l'achat des animaux et la Société Agroalimentaire Sahel Vert de Zinder pour la disponibilité du tourteau de Balanites aegyptiaca.

\section{REFERENCES}

Ahmad E, Ahmad N, Naseer Z, Aleem M, Khan MS, Ashiq M, Younis M. 2011. Relationship of age to body weight, scrotal circumference, testicular ultrasonograms, and semen quality in Sahiwal bulls. Trop. Anim. Health Prod., 43:159-164. DOI 10.1007/s11250-0109668-1

Elhadji Nouhou M. 2014. Effets d'une substitution du tourteau de graines de coton par les gousses de Piliostigma reticulatum (De.Candolle) Hochstetter dans l'alimentation, sur les performances de croissance de la chèvre rousse de Maradi. Mémoire de Master : Productions Animales et Développement Durable, EISMV de l'Université Cheikh Anta Diop, Dakar, 40p.

FAO. 2018. Statistiques des animaux domestiques.

FAO. http://www.fao.org/faostat/en/\#data/QA. Consulté le 04 juin 2019.

Gnanda IB, Bougouma-Yameogo MCV, Wereme N'diaye A, Ouedraogo $\mathrm{T}$, 
Kabore A, Lodoun B, Sinon B. 2015a. L'embouche bovine dans les élevages du Plateau Central du Burkina Faso: Résultats économiques d'une démarche de validation d'un référentiel technicoéconomique sur la spéculation. Int. J. Biol. Chem. Sci., 9(6): 2648-2662. DOI: http://dx.doi.org/10. 4314/ijbcs.v9i6.11.

Gnanda IB, Nignan M, Ouedraogo S, Wereme N'diaye A, Traore O, Sinon B. 2015b. Influence d'une co-construction de rationnement amélioré sur les performances d'embouche ovine paysanne dans la commune rurale de Korsimoro au Burkina Faso. Int. J. Biol. Chem. Sci., 9(3): 1544-1556. DOI: http://dx.doi.org/10.4314/ijbcs.v9i3.35.

Kiema A, Nianogo AJ, Ouedraogo T, Somda J. 2008. Valorisation des ressources alimentaires locales dans l'embouche ovine paysanne: performances technicoéconomiques et option de diffusion. Cahiers Agricultures, 17(1): 23 - 27. DOI: 10.1684/agr.2008.0154.

Malam Abdou M, Salissou I, Abdou D, Germain JS. 2017b. Analyse tecnicoéconomique des aliments densifiés sur les performances de croissance de cabris roux de Maradi au Niger. Int. J. Biol. Chem. Sci., 11(1): 280-292. DOI: http://dx.doi.org/10.4314/ijbcs.v11i1.22

Malam Abdou M, Salissou I, Dan Gomma A, Sow A, Sawadogo GJ. 2017a. Estimation des rendements et de la rentabilité économique de production de trois cultures : le sorgho, le niébé et la dolique à Djirataoua (Maradi - République du Niger). Journal of Applied Biosciences, 117:11642-11650.

DOI: https://dx.doi.org/10.4314/jab.v117i1.1.

Mohamed AM, Wolf W, Well S. 2002. Physical, morphological and chemical characteristics, oil recovery and fatty acid composition of Balanites aegyptiaca Kernels. Plant Foods Hum. Nutr., 57: 179-89. DOI:10.1023/a: 1015237612018

Moxon R, Bright L, Pritchard B, Bowen IM, de Souza MB, da Silva LD, England GC. 2015. Digital image analysis of testicular and prostatic ultrasonographic echogencity and heterogeneity in dogs and the relation to semen quality. Anim. Reprod. Sci., 160: 112-9. DOI: http://dx.doi.org/10.1016/j.anireprosci.20 15.07.012

Safoura O. 2006. Potentialités fourragères et essais d'amélioration de la valeur nutritive de trois ligneux fourragers : Piliostigma thonningii Schumach Mile Redh, Piliostigma reticulatum(D.C.) Hoscht et Khaya senegalensis (Desr.) A. Juss. Mémoire d'Ingénieur du Developpement Rural/Option, Université Polytechnique de Bobo Dioulasso, 67 p.

Soumana I, Ayssiwede SB, Issa S, Guero, Missohou A. 2016. Effet de la complémentation avec des blocs multinutritionnels concentrés à base de Sida cordifolia L., une plante invasive, sur les performances zootechnicoéconomiques des antenais de race Balami à Déréké/Dosso (Niger). International Journal of Innovation and Applied Studies, 15(3): 716-730. http://www.ijias.issr-journals.org/.

Tiétiambou FRS, Bazougo P, Diallo ADA, Kouyaté AM, Lykke AM, Bassolé IHN, Ouédraogo A. 2015. Production de l'huile de Balanites aegyptiaca. Fiche technique, Projet QualiTree, Université de Ouagadougou, 2p.

Zoundi JS, Sawadogo 1, Nianogo AJ. 2005. Utilisation de blocs multi nutritionnels en substitution partielle de concentré pour l'engraissement des ovins au sein des systèmes mixtes agriculture-élevage du plateau central du Burkina Faso. Journal des Sciences, 5 (1):15-22. 